RESEARCH BRIEF

\title{
Smoke-Free Rules in Homes and Cars Among Smokers and Nonsmokers in Minnesota
}

\author{
Michael J. Parks, $\mathrm{PhD}^{1,2}$; John H. Kingsbury, $\mathrm{PhD}^{3}$; Raymond G. Boyle, PhD, MPH${ }^{4}$; \\ Sharrilyn Evered, $\mathrm{PhD}^{5}$
}

Accessible Version: www.cdc.gov/pcd/issues/2018/17_0355.htm

Suggested citation for this article: Parks MJ, Kingsbury JH, Boyle RG, Evered S. Smoke-Free Rules in Homes and Cars Among Smokers and Nonsmokers in Minnesota. Prev Chronic Dis 2018;15:170355. DOI: https://doi.org/10.5888/pcd15.170355.

\section{PEER REVIEWED}

\section{Abstract}

We examined prevalence and predictors of comprehensive smokefree household rules (ie, smoke-free homes and cars) among smokers and nonsmokers in Minnesota. Data came from the 2014 Minnesota Adult Tobacco Survey; weighted analyses consisted of descriptive analyses and multivariate logistic regression analyses. Most adult smokers implemented home-only smoke-free rules (43\%) while most nonsmokers implemented comprehensive smoke-free rules (home and car; 85\%). Comprehensive smokefree rules were more common among people with high socioeconomic status (SES), married people, and people who did not live with a smoker; those with a child in the home were more likely to implement smoke-free homes but not smoke-free cars. Public health practitioners should focus on addressing the majority of smokers who do not implement comprehensive smoke-free household rules, such as low-SES populations, and addressing caregivers who do not implement smoke-free car rules.

\section{Objective}

Smoke-free public policies reduce secondhand smoke in public places; however, private spaces, such as the home and car, are common locations for secondhand smoke exposure (1). Voluntary smoke-free rules in homes can reduce secondhand smoke and tobacco use, especially among households with children (2-4). Smoke-free homes have become more prevalent, but disparities persist $(2,5-7)$. Limited information exists on comprehensive smoke-free rules (ie, smoke-free homes and cars) $(2,7,8)$, which are optimal, particularly for children $(2,9)$. We examined prevalence and predictors of smoke-free rules among smokers and nonsmokers in Minnesota.

\section{Methods}

Data came from the 2014 Minnesota Adult Tobacco Survey (MATS). MATS is a statewide, cross-sectional landline and cellular telephone survey of 9,304 Minnesotans aged 18 years or older, which yielded a combined response rate of $71 \%$. MATS was approved by Minnesota Department of Health's Institutional Review Board. Survey weights accounted for sampling and ensured statewide representativeness. Smoke-free rules were measured with 2 questions: 1) "Which statement best describes rules about smoking inside your home (excluding porches and garages): not allowed anywhere, allowed some places or at some times, or allowed anywhere"; and 2) "In the vehicles (excluding motorcycles) that you or your family who live with you own or lease, is smoking ... allowed, sometimes allowed in at least one vehicle, or never allowed in any vehicle." A composite, 4-category variable captured smoke-free rules: comprehensive (smoke-free home and car), home-only (smoke-free home, not car), car-only (smoke-free car, not home), and no rules (smoking allowed in home and car). Covariates were age (4 categories), education (4 categories), lowincome (yes or no), race/ethnicity (white or other), sex (male or female), marital status (married or not married), living with a child aged less than 18 years (yes or no), location (metropolitan county or nonmetropolitan county), and living with a smoker (yes or no). We used a 3-category measure of smoking intensity: light, moderate, and heavy.

Descriptive analyses were used to compare demographic characteristics and smoking behaviors across different household smokefree rules for smokers and nonsmokers separately. All analyses were conducted using the svyset command in Stata, version 13 
(StataCorp LLC). Stata's default of $F$ ratios and adjusted Wald tests were used to compare continuous variables across smoke-free rules categories; $\chi^{2}$ statistics and design-adjusted $F$ ratios were used to compare binary variables. A multivariate logistic regression was used to assess characteristics associated with comprehensive smoke-free rules for smokers and nonsmokers separately.

\section{Results}

Most adult smokers implemented home-only smoke-free rules (43\%) or did not implement any smoke-free rules (31\%) (Figure). A smaller proportion of smokers implemented comprehensive smoke-free rules (home and car) (19\%). Most nonsmokers implemented comprehensive smoke-free rules (85\%) (Figure). There were significant differences in demographics and behaviors across different smoke-free rules among smokers and nonsmokers (Table).

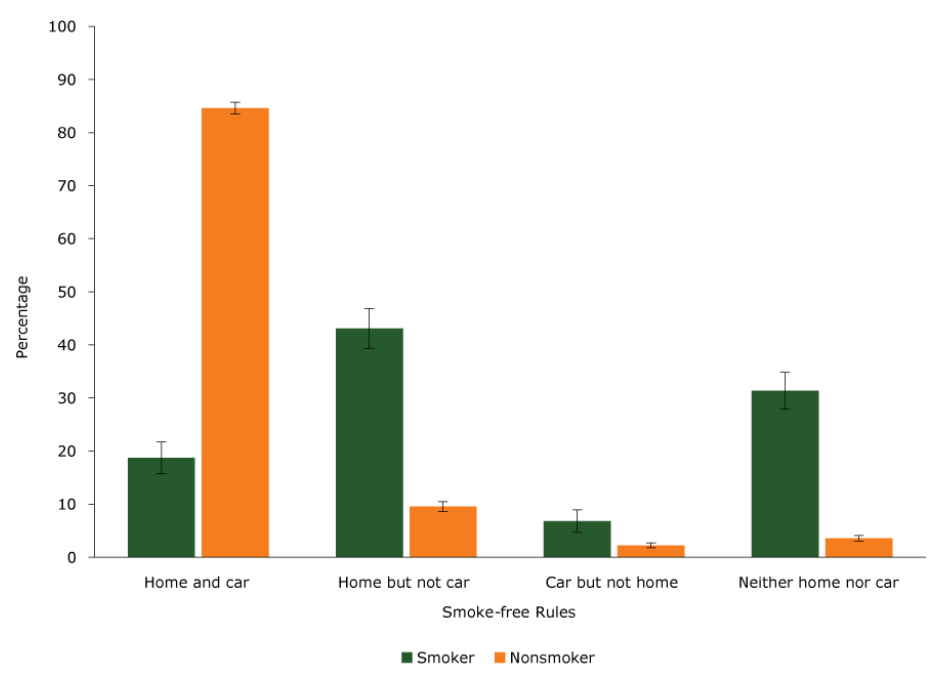

Figure. Percentage of smokers and nonsmokers who implemented voluntary smoke-free rules in the home and car, Minnesota Adult Tobacco Survey, 2014. Error bars indicate 95\% confidence intervals.

Multivariate logistic regressions showed that income, marital status, and smoking intensity were related to comprehensive smoke-free rules among smokers. The odds of implementing comprehensive rules decreased for moderate and heavy smokers compared with light smokers (adjusted odds ratio [AOR] for moderate smokers, 0.13 ; 95\% CI, 0.07-0.23; and AOR for heavy smokers, $0.21 ; 95 \%$ CI, 0.06-0.71). The odds of implementing comprehensive rules increased for married smokers compared with unmarried smokers (AOR, 1.80; 95\% CI, 1.09-2.98). Low-income status was negatively associated with implementing comprehensive rules (AOR, 0.57; 95\% CI, 0.34-0.94). Having a child in the home aged less than 18 years had a nonsignificant association with comprehensive rules for smokers (supplementary analyses showed a significant association with smoke-free home rules).

For nonsmokers, men were less likely than women to implement comprehensive smoke-free rules (AOR, 0.75; 95\% CI, 0.61-0.92). Higher education levels were positively related to comprehensive rule implementation (AOR, 1.30; 95\% CI, 1.15-1.48), and low-income status was negatively related to comprehensive rule implementation (AOR, 0.75; 95\% CI, 0.59-0.97). Married nonsmokers were more likely to implement comprehensive rules than were unmarried nonsmokers (AOR, 1.98; 95\% CI, 1.58-2.50), and the odds of implementing comprehensive rules were lower for nonsmokers who lived with a smoker than with those who did not (AOR, 0.08; 95\% CI, 0.07-0.11). Having a child in the home under 18 years had a nonsignificant association with implementing comprehensive smoke-free rules for nonsmokers.

\section{Discussion}

This study adds to the literature on voluntary, smoke-free rules by establishing the prevalence and predictors of comprehensive smoke-free rules (ie, in homes and cars). We found marked disparities in comprehensive smoke-free rule implementation across smoking status and key demographics.

Although $85 \%$ of nonsmokers implemented comprehensive rules, only $19 \%$ of smokers implemented such comprehensive rules, and $43 \%$ implemented home-only rules. Previous research has documented an increase in smoke-free rules in homes (2), indicating that the general public understands the harm of secondhand smoke in homes. However, we found that smoking in the car was common, even among smokers who implement a smoke-free home.

People with low income and low education levels were less likely to implement comprehensive smoke-free rules. Practitioners should focus on reducing SES-related barriers to implementing comprehensive smoke-free rules (eg, health care access, "knowledge gaps" of the danger of secondhand smoke). Tailored health education efforts regarding comprehensive smoke-free rules may help to address these disparities. Living with a smoker was also a barrier to implementation of smoke-free rules (among smokers and nonsmokers); consequently, programs should communicate the importance of comprehensive smoke-free rules by addressing the entire household.

Living with a child under age 18 years predicted smoke-free home rules, supporting previous research $(2,10)$, but living with a child did not predict smoke-free car rules for most smokers. Private

\footnotetext{
The opinions expressed by authors contributing to this journal do not necessarily reflect the opinions of the U.S. Department of Health and Human Services, the Public Health Service, the Centers for Disease Control and Prevention, or the authors' affiliated institutions.
} 
spaces, such as homes and cars, are major sources of secondhand smoke exposure, particularly for children (1). Implementing less than comprehensive smoke-free rules increases the risk of secondhand smoke exposure, and voluntary rules can protect children against secondhand smoke in private spaces that are typically unaffected by public policy $(3,11)$. Given the dangers associated with secondhand smoke exposure in confined spaces such as cars (12), public health programs, media campaigns, and primary care interventions should promote comprehensive smoke-free rules among smokers, and particularly among caregivers and parents who implement less than comprehensive rules $(3,9)$.

\section{Acknowledgments}

This research was funded by ClearWay Minnesota, an independent nonprofit organization, the Statewide Health Improvement Program (SHIP), and State Core Tobacco Control funding from the Centers for Disease Control and Prevention, CDC-RFA-DP151509 (1U58DP006005-01). SHIP and the Centers for Disease Control and Prevention had no role in the study design, data collection, analysis, interpretation of the data, writing of the article, or decision to submit for publication.

\section{Author Information}

Corresponding Author: Michael J. Parks, PhD, Health Promotion and Chronic Disease Division, Minnesota Department of Health, 85 E 7th Place, St Paul, MN 55164. Telephone: 651-201-5285. Email: michael.parks@state.mn.us.

Author Affiliations: ${ }^{1}$ Department of Pediatrics, Medical School, University of Minnesota, St Paul, Minnesota. ${ }^{2}$ Health Promotion and Chronic Disease Division, Minnesota Department of Health, St Paul, Minnesota. ${ }^{3}$ Office of Statewide Health Improvement Initiative, Minnesota Department of Health, St Paul, Minnesota. ${ }^{4}$ Department of Research Programs, ClearWay Minnesota, Minneapolis, Minnesota. ${ }^{5}$ Center for Health Statistics, Minnesota Department of Health, St Paul, Minnesota.

\section{References}

1. King BA, Patel R, Babb SD. Prevalence of smokefree home rules - United States, 1992-1993 and 2010-2011. MMWR Morb Mortal Wkly Rep 2014;63(35):765-9.

2. King BA, Patel R, Babb SD, Hartman AM, Freeman A. National and state prevalence of smoke-free rules in homes with and without children and smokers: two decades of progress. Prev Med 2016;82:51-8.
3. Nicholson JS, McDermott MJ, Huang Q, Zhang H, Tyc VL. Full and home smoking ban adoption after a randomized controlled trial targeting secondhand smoke exposure reduction. Nicotine Tob Res 2015;17(5):612-6.

4. Keyes M, Legrand LN, Iacono WG, McGue M. Parental smoking and adolescent problem behavior: an adoption study of general and specific effects. Am J Psychiatry 2008; 165(10):1338-44.

5. St Claire AW, Boyle RG, Schillo BA, Rode P, Taylor KA. Smokefree home rules adoption by smokers and nonsmokers: Minnesota, 1999-2010. Am J Prev Med 2012;43(5,Suppl 3):S197-204.

6. Hawkins SS, Berkman L. Parental home smoking policies: the protective effect of having a young child in the household. Prev Med 2011;53(1-2):61-3.

7. Norman GJ, Ribisl KM, Howard-Pitney B, Howard KA. Smoking bans in the home and car: do those who really need them have them? Prev Med 1999;29(6 Pt 1):581-9.

8. Binns HJ, O’Neil J, Benuck I, Ariza AJ; Pediatric Practice Research Group. Influences on parents' decisions for home and automobile smoking bans in households with smokers. Patient Educ Couns 2009;74(2):272-6.

9. Nabi-Burza E, Regan S, Drehmer J, Ossip D, Rigotti N, Hipple $\mathrm{B}$, et al. Parents smoking in their cars with children present. Pediatrics 2012;130(6):e1471-8.

10. Borland R, Yong H-H, Cummings KM, Hyland A, Anderson $\mathrm{S}$, Fong GT. Determinants and consequences of smoke-free homes: findings from the International Tobacco Control (ITC) Four Country Survey. Tob Control 2006;15(Suppl 3):iii42-50.

11. Jarvie JA, Malone RE. Children's secondhand smoke exposure in private homes and cars: an ethical analysis. Am J Public Health 2008;98(12):2140-5.

12. Bohac DL, Waldhart E, Zhou Z, Hewett M. Personal vehicle secondhand smoke exposure for various ventilation modes. Tob Regul Sci 2017;3(4):492-503.

The opinions expressed by authors contributing to this journal do not necessarily reflect the opinions of the U.S. Department of Health and Human Services, the Public Health Service, the Centers for Disease Control and Prevention, or the authors' affiliated institutions. 


\section{Table}

Table. Smoke-Free Rules in the Home and Car and Comparative Characteristics Among Minnesota Adult Smokers and Nonsmokers, Minnesota Adult Tobacco Survey, $2014^{\mathrm{a}}$

\begin{tabular}{|c|c|c|c|c|c|}
\hline Variable $^{b}$ & Home and Car & Home But Not Car & Car But Not Home & Neither Home Nor Car & $P$ Value $^{c}$ \\
\hline \multicolumn{6}{|c|}{ Smokers } \\
\hline \multicolumn{6}{|l|}{ Age, $y$} \\
\hline $18-24$ & $24.6(0.34)$ & $45.4(0.39)$ & $04.0(0.15)$ & $26.0(0.35)$ & $<.001$ \\
\hline $25-44$ & $19.5(0.32)$ & $54.7(0.40)$ & $03.0(0.14)$ & $22.9(0.34)$ & $<.001$ \\
\hline $45-64$ & $16.3(0.38)$ & $32.3(0.48)$ & $12.5(0.34)$ & $38.9(0.50)$ & $<.001$ \\
\hline$\geq 65$ & $16.9(0.50)$ & $13.6(0.46)$ & $10.0(0.40)$ & $59.7(0.66)$ & $<.001$ \\
\hline White (vs other) & $17.9(0.37)$ & $44.2(0.47)$ & $05.3(0.21)$ & $32.5(0.45)$ & .03 \\
\hline Male (vs female) & $19.5(0.35)$ & $44.1(0.44)$ & $05.6(0.20)$ & $30.8(0.41)$ & .57 \\
\hline Metropolitan county (vs nonmetropolitan county) & $20.8(0.32)$ & $42.8(0.39)$ & $08.3(0.22)$ & $28.1(0.35)$ & .03 \\
\hline Education $^{\mathrm{d}}$, category mean (SD) & $02.6(0.78)$ & $02.6(0.72)$ & $02.1(0.70)$ & $02.3(0.86)$ & $<.001$ \\
\hline Low income ${ }^{\mathrm{e}}$ (vs other) & $12.0(0.32)$ & $33.5(0.47)$ & $09.9(0.30)$ & $44.6(0.50)$ & $<.001$ \\
\hline Married (vs unmarried) & $22.5(0.38)$ & $43.8(0.45)$ & $05.9(0.21)$ & $27.8(0.41)$ & .18 \\
\hline Child aged $<18 \mathrm{y}$ in household & $21.1(0.34)$ & $53.8(0.41)$ & $01.9(0.12)$ & $23.2(0.35)$ & $<.001$ \\
\hline Lives with smoker & $14.7(0.31)$ & $40.1(0.43)$ & $08.2(0.24)$ & $36.9(0.42)$ & $<.01$ \\
\hline \multicolumn{6}{|l|}{ Smoking intensity ${ }^{f}$} \\
\hline Light & $25.7(0.40)$ & $45.9(0.45)$ & $06.8(0.23)$ & $21.6(0.37)$ & $<.001$ \\
\hline Moderate & $05.9(0.22)$ & $39.7(0.47)$ & $06.9(0.24)$ & $47.5(0.48)$ & $<.001$ \\
\hline Heavy & $06.0(0.25)$ & $36.1(0.51)$ & $07.6(0.28)$ & $50.3(0.53)$ & $<.001$ \\
\hline \multicolumn{6}{|c|}{ Nonsmokers } \\
\hline \multicolumn{6}{|l|}{ Age, y } \\
\hline $18-24$ & $71.5(0.35)$ & $21.6(0.32)$ & $02.9(0.13)$ & $04.0(0.15)$ & $<.001$ \\
\hline $25-44$ & $87.4(0.28)$ & $09.5(0.25)$ & $01.6(0.10)$ & $01.5(0.10)$ & $<.001$ \\
\hline $45-64$ & $84.8(0.37)$ & $07.9(0.28)$ & $02.1(0.15)$ & $05.2(0.23)$ & $<.001$ \\
\hline$\geq 65$ & $87.4(0.44)$ & $05.1(0.29)$ & $03.5(0.24)$ & $04.0(0.26)$ & $<.001$ \\
\hline White (vs other) & $85.0(0.37)$ & $09.3(0.30)$ & $02.1(0.15)$ & $03.6(0.19)$ & .21 \\
\hline Male (vs female) & $82.9(0.36)$ & $10.6(0.30)$ & $02.3(0.15)$ & $04.2(0.19)$ & .02 \\
\hline Metropolitan county (vs nonmetropolitan county) & $84.5(0.31)$ & $09.7(0.26)$ & $02.3(0.13)$ & $03.5(0.16)$ & .86 \\
\hline Education $^{\mathrm{d}}$, category mean (SD) & $03.0(0.91)$ & $02.8(0.76)$ & $02.7(1.10)$ & $02.6(0.82)$ & $<.001$ \\
\hline Low income ${ }^{\mathrm{e}}$ (vs other) & $77.6(0.01)$ & $13.4(0.01)$ & $03.1(0.01)$ & $05.8(0.01)$ & $<.001$ \\
\hline
\end{tabular}

${ }^{a}$ All estimates were derived by using survey weights.

${ }^{\mathrm{b}}$ All values are \% (SD) except where otherwise noted.

${ }^{c} F$ ratios and adjusted Wald tests were used to compare continuous variables; $x^{2}$ statistics and design-adjusted $F$ ratios were used to compare binary variables.

${ }^{d}$ Education was a 4-category measure: 1 = no high school degree; 2 = high school degree; $3=$ more than high school degree but no college degree; and $4=$ college degree or more.

${ }^{\mathrm{e}}$ Low income was defined as the lowest $25 \%$ of the distribution of our income measure, which was equivalent to less than $\$ 30,000$ total household income per year.

${ }^{f}$ Smoking intensity is based on smoking frequency and number of cigarettes per day (light $=<15$ cigarettes per day and both daily and nondaily smoker; moderate $=>15$ but $<25$ cigarettes per day and a daily smoker; heavy $=>25$ cigarettes per day and a daily smoker.

The opinions expressed by authors contributing to this journal do not necessarily reflect the opinions of the U.S. Department of Health and Human Services, the Public Health Service, the Centers for Disease Control and Prevention, or the authors' affiliated institutions. 
(continued)

Table. Smoke-Free Rules in the Home and Car and Comparative Characteristics Among Minnesota Adult Smokers and Nonsmokers, Minnesota Adult Tobacco Survey, $2014^{a}$

\begin{tabular}{|c|c|c|c|c|c|}
\hline Variable $^{\mathrm{b}}$ & Home and Car & Home But Not Car & Car But Not Home & Neither Home Nor Car & $P$ Value $^{c}$ \\
\hline Married (vs unmarried) & $88.9(0.01)$ & $06.4(0.01)$ & $01.8(0.00)$ & $02.9(0.00)$ & $<.001$ \\
\hline Child aged $<18 \mathrm{y}$ in household & $87.0(0.01)$ & $09.9(0.01)$ & $01.1(0.00)$ & $02.0(0.00)$ & $<.001$ \\
\hline Lives with smoker & $44.2(0.02)$ & $34.8(0.02)$ & $04.1(0.01)$ & $16.9(0.02)$ & $<.001$ \\
\hline
\end{tabular}

${ }^{a}$ All estimates were derived by using survey weights.

${ }^{\mathrm{b}}$ All values are \% (SD) except where otherwise noted.

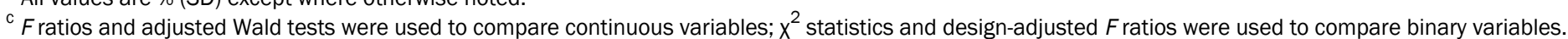

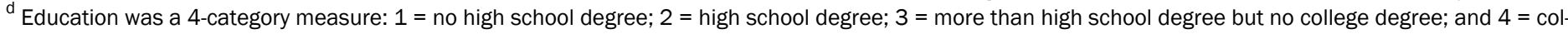
lege degree or more.

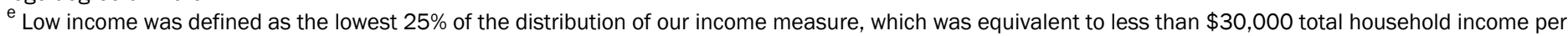
year.

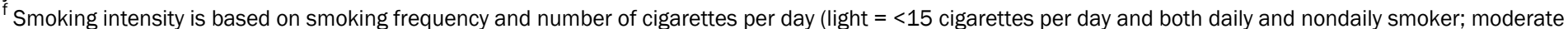
$=>15$ but $<25$ cigarettes per day and a daily smoker; heavy $=>25$ cigarettes per day and a daily smoker.

The opinions expressed by authors contributing to this journal do not necessarily reflect the opinions of the U.S. Department of Health and Human Services, the Public Health Service, the Centers for Disease Control and Prevention, or the authors' affiliated institutions. 